\title{
Peroxisome Proliferator-Activated Receptor-Gamma Reduces ER Stress and Inflammation via Targeting NGBR Expression
}

\begin{abstract}
Jialing Ma ${ }^{1}$, Peng Zeng ${ }^{2}$, Lipei Liu ${ }^{2}$, Mengmeng Zhu ${ }^{1}$, Juan Zheng ${ }^{1}$, Chengyi Wang ${ }^{1}$, Xiaokang Zhao', Wenquan $\mathrm{Hu}^{3}$, Xiaoxiao Yang ${ }^{1}$, Yajun Duan ${ }^{1}$, Jihong Han ${ }^{1,2}$, Qing R. Miao ${ }^{4}$ and Yuanli Chen ${ }^{1 *}$

${ }^{1}$ Key Laboratory of Major Metabolic Diseases and Nutritional Regulation of Anhui Department of Education, College of Food and Biological Engineering, Hefei University of Technology, Hefei, China, ${ }^{2}$ College of Life Sciences, State Key Laboratory of Medicinal Chemical Biology, Key Laboratory of Bioactive Materials of Ministry of Education, Nankai University, Tianjin, China, ${ }^{3}$ Centre for Lipid Research \& Key Laboratory of Molecular Biology for Infectious Diseases (Ministry of Education), Chongqing Medical University, Chongqing, China, ${ }^{4}$ Diabetes and Obesity Research Center, New York University Long Island School of Medicine, New York, NY, United States
\end{abstract}

Increased Nogo-B receptor (NGBR) expression in the liver improves insulin sensitivity by reducing endoplasmic reticulum stress (ER stress) and activating the AMPK pathway, although it remains elusive the mechanisms by which NGBR is induced. In this study, we found that PPAR $\gamma$ ligands (rosiglitazone or pioglitazone) increased NGBR expression in hepatic cells and HUVECs. Furthermore, promoter analysis defined two PPREs (PPAR $\gamma$ responsive elements) in the promoter region of NGBR, which was further confirmed by the ChIP assay. In vivo, using liver-specific PPAR $y$ deficient $\left(P P A R \gamma^{L K O}\right)$ mice, we identified the key role of PPAR $y$ expression in pioglitazone-induced NGBR expression. Meanwhile, the basal level of ER stress and inflammation was slightly increased by NGBR knockdown. However, the inhibitory effect of rosiglitazone on inflammation was abolished while rosiglitazone-inhibited ER stress was weakened by NGBR knockdown. Taken together, these findings show that NGBR is a previously unrecognized target of PPAR $y$ activation and plays an essential role in PPAR $\gamma$-reduced ER stress and inflammation.

Keywords: PPAR $\gamma$, rosiglitazone, NGBR, ER stress, inflammation

\section{INTRODUCTION}

The endoplasmic reticulum (ER) is an essential multifunctional organelle, which is the primary place for protein synthesis, folding, and assembly, as well as degradation of misfolded or damaged proteins. When ER homeostasis is disrupted, misfolded proteins gather in the ER and subsequently activate the unfolded protein response (UPR) (Walter and Ron, 2011). After activation, UPR will reconstruct

\footnotetext{
Abbreviations: AKT, Protein kinase B; AMPK, adenosine monophosphate activated protein kinase; ATF6, activating transcription factor 6; BIP, binding immunoglobulin protein; Chop, C/EBP homologous protein; ER stress, endoplasmic reticulum stress; LPS, Lipopolysaccharide; NGBR, Nogo-B receptor; NF-кB, nuclear factor $\kappa B$; PPAR $\gamma$, Proliferator-activated receptor gamma; p-IRE1 $\alpha$, phosphorylated inositol-requiring enzyme $1 \alpha$; p-PERK, phosphorylated protein kinase RNA-dependent ER kinase; TNF- $\alpha$, tumor necrosis factor alpha; IL-1 $\beta$, interleukin 1 beta.
} 
cellular transcription and translation, as well as degradation pathways to resolve defects in protein folding. These effects are accomplished by activating three transmembrane ER proteins, namely protein kinase RNA-dependent ER kinase (PERK), inositol-requiring enzyme 1 (IRE-1), and activating transcription factor 6 (ATF6) (Hetz, 2012). ER stress links to many inflammatory and stress signaling, including the activation of the nuclear factor $\kappa \mathrm{B}$ (NF- $\kappa \mathrm{B}$ ) pathway (Kharroubi et al., 2004). Both inflammation and ER stress are short-term adaptive systems that are indispensable for the function and survival of the organism (Hotamisligil, 2010). However, they are detrimental when the inflammation and ER stress are chronically engaged (Hotamisligil, 2010; Fu et al., 2012). ER stress and chronic inflammation are related to many diseases, including diabetes, obesity, neurodegenerative and neuromuscular inflammatory diseases (Xue et al., 2016). ER stress can strengthen many stress and inflammatory signal pathways, aggravate metabolic disorders, and lead to obesity, insulin resistance, NAFLD, and dyslipidemia (Wang and Kaufman, 2012).

On the one hand, Nogo-B receptor (NGBR) was identified as a receptor for Nogo-B in human umbilical vein endothelial cells (HUVECs). Restraining NGBR expression can eliminates NogoB-mediated endothelial cell (EC) migration, attenuates vascular endothelial growth factor (VEGF)-stimulated phosphorylation of Protein kinase B (Akt) and HUVECs chemotaxis and morphogenesis (Miao et al., 2006). On the other hand, NGBR can independently affect nervous system regeneration. Moreover, NGBR can promote Niemann-Pick type C2 protein (NPC2)dependent cholesterol trafficking and $\mathrm{AMPKa}$-liver $\mathrm{X}$ receptor (LXR)-dependent free fatty acids (FFA) and triglycerides (TG) metabolism ( $\mathrm{Hu}$ et al., 2016; Zhang et al., 2018). NGBR may promote or inhibit the occurrence and progression of tumor, which depends on the types of tumors (Wu et al., 2018). Our previous studies demonstrate that overexpression of NGBR improves glucosamine hydrochloride (Glcn)-induced ER stress. Likewise, overexpression of NGBR in the liver reduced ER stress and increased insulin sensitivity in high-fat diet (HFD)/streptozotocin (STZ)-treated mice tissues, suggesting that hepatic NGBR may be necessary to maintain insulin sensitivity and prevent type 2 diabetes (T2D) (Chen et al., 2021).

Three subtypes of peroxisomal proliferator-activated receptors (PPARs) have been identified: PPARa, $\beta$ and $\gamma$, which are all ligand-activated nuclear transcriptional factors. PPAR and retinoid $\mathrm{X}$ receptor (RXR) can form a heterodimer to bind to a DNA recognition motif named PPAR response element (PPRE), which consists of a direct repeat separated by one nucleotide (also known as DR1) with a consensus sequence of AGGTGA-X-AGGTCA. Synthetic PPAR $\gamma$ agonists, including rosiglitazone and pioglitazone, effectively enhance insulin sensitivity and are currently used as drugs for T2D (Huang et al., 2021). Studies have demonstrated that ER stress and inflammatory responses are downregulated after treatment with various PPARy agonists (Ricote et al., 1998; Straus and Glass, 2007; Yoshiuchi et al., 2009) across multiple cell types and tissues. However, the mechanisms underlying PPAR $\gamma$-inhibited ER stress and inflammation are not fully understood.

Considering the functions of NGBR in EC and liver, which all contribute to insulin sensitivity and inflammation, we determined the effect of PPAR $\gamma$ activation on NGBR expression and function in vitro with EC and hepatocytes and in vivo. We proved that NGBR was a previously unrecognized target of PPAR $\gamma$. Besides, we also tried to reveal that PPAR $\gamma$ ligand-alleviated inflammation and ER stress were related to NGBR expression.

\section{MATERIALS AND METHODS}

\section{Reagents}

Rosiglitazone, pioglitazone and GW9662 were purchased from Cayman Chemical (Ann Arbor, MI, United States). LPS and tunicamycin were purchased from Sigma-Aldrich (St. Louis, MO, United States). Rabbit anti-NGBR, $\beta$-actin, and GAPDH polyclonal antibodies were purchased from Abcam (Cambridge, MA, United States). Rabbit anti-IL-1 $\beta$ and IL-6 polyclonal antibodies were purchased from ABclonal (Wuhan, China). Rabbit antiPPAR $\gamma$ and AKT polyclonal antibodies and mouse anti-TNF- $\alpha$ and phospho-AKT ( $p$-AKT, Ser473) monoclonal antibodies were purchased from Proteintech Group (Chicago, IL, United States). Rabbit anti-CHOP, BIP, ATF6, phospho-IRE1a (p-IRE1a, Ser724) and phospho-PERK (p-PERK, Thr982) polyclonal antibodies were purchased from Affinity Biosciences (Cincinnati, $\mathrm{OH}$, United States). Silencer siRNA Construction Kit was purchased from Life Technologies (New York, United States). Transfection reagent Lipofectamine RNAiMAX and Lipofectamine 2000 were purchased from Thermo Fisher Scientific (Waltham, MA, United States).

\section{Cell Culture}

HUVEC (human umbilical vein endothelial cell), HepG2 (human hepatoma cell line), and 293T (human embryonic kidney cell) were purchased from ATCC (Manassas, VA, United States) and cultured in DMEM containing 10\% fetal bovine serum (FBS), $50 \mu \mathrm{g} / \mathrm{ml}$ of Pen-Strep and $2 \mathrm{mM}$ glutamine. Cells received treatment at $80-90 \%$ confluence. Mouse primary hepatocytes were isolated from PPAR $\gamma^{\text {flox/flox }}$ and liver-specific PPAR $\gamma$ knockout $\left(\mathrm{PPAR} \gamma^{\mathrm{LKO}}\right)$ mice by a collagenase perfusion method as described (Zhang et al., 2018).

\section{Western Blotting}

After treatment, total cellular proteins were extracted from primary hepatocytes, HUVEC, HepG2 cells, or a piece of mouse liver, then the expression of PPAR $\gamma, \mathrm{NGBR}, \mathrm{AKT}$, $p$-AKT, CHOP, BIP, IL-1 $\beta$, TNF- $\alpha$, NF- $\kappa$ B, ATF6, p-PERK, p-IRE1a protein was detected by Western blotting (Chen et al., 2012). After capturing the images, all bands from three repeated experiments were quantified with statistical analysis by two technicians who were blinded to the treatments.

\section{SiRNA Transfection}

HepG2 cells or HUVECs were divided into a six-well culture plate. After reaching 30-40\% confluence, cells were transfected with negative control siRNA (si-NC) or NGBR siRNA (siNGBR) using Lipofectamine RNAiMAX (Duan et al., 2012). After $24 \mathrm{~h}$ transfection, the cells were treated with rosiglitazone $(10 \mu \mathrm{M})$ for $12 \mathrm{~h}$, then treated with LPS $(100 \mathrm{ng} / \mathrm{ml})$ with or without rosiglitazone for another $12 \mathrm{~h}$. The corresponding protein expression was determined. 
TABLE 1 | The sequences of primers for $\mathrm{qPCR}$ analysis.

\begin{tabular}{|c|c|c|}
\hline Gene & Forward & Reverse \\
\hline h-NGBR & AGCCTCGTGGTGTGGTGTA & GCCCAGAAGTTCTTGCTGTT \\
\hline h-GAPDH & GGTGGTCTCCTCTGACTTCAACA & GTTGCTGTAGCCAAATTCGTT \\
\hline h-PPAR $\gamma$ & TCAAAGGAGTGGGAGTGGTC & CAAGGCCATITCTCAAACG \\
\hline h-CHOP & GGAAACAGAGTGGTCATTCCC & СTGCTTGAGCCGTTCATTCTC \\
\hline h-BIP & TTGACTCCGACCTTCACCTTCC & TाTCACAGTGGCCAAGAGTC \\
\hline$h-T N F-a$ & CGTCGTAGCAAACCACCAAG & TTGAAGAGAACCTGGGAGTAGACA \\
\hline $\mathrm{h}-\mathrm{IL}-1 \beta$ & GACCTTCCAGGATGAGGACA & AGCTCATATGGGTCCGACAG \\
\hline $\mathrm{h}-\mathrm{NF}-\kappa \mathrm{B}$ & GCTCTTACTGACTGGCATGAG & CGCAGCTCTAGGAGCATGTG \\
\hline m-NGBR & GAGGAAGCCCACAGATCTGGATGTA & TCTGATITGCCAGGGAAGAAAGCC \\
\hline m-GAPDH & ACCCAGAAGACTGTGGATGG & ACACATTGGGGGTAGGAACA \\
\hline
\end{tabular}

\section{Real-Time Quantitative RT-PCR (qPCR)}

The total RNApure reagent (Zomanbio, Beijing, China) was used to extract total RNA from cells or a piece of the liver. Then, $1 \mu \mathrm{g}$ total RNA from each sample was reverse-transcribed into cDNA using a reverse transcription kit, followed by quantitative PCR according to the manufacturer's instructions for SYBR Green Master Mix (Bio-Rad) and the primers were listed in Table $\mathbf{1 .}$ GAPDH or $\beta$-actin were used for normalization.

\section{NGBR Luciferase Reporter Assay}

PPAR $\gamma$ overexpression plasmid was constructed as described (Duan et al., 2012). Human NGBR promoter (from -983 to +167, pNGBR) sequence was amplified using PCR technic with genomic DNA (extracted from HepG2) and the following primers: forward, $5^{\prime}$-TGCACTCGAGGATGATAGAGGATG TA- $3^{\prime}$ and reverse, $5^{\prime}$-TGCCAAGCTTACTCTTGTGGCCCT C-3'. After confirming the sequence, the PCR product was double digested and ligated into the pGL4 luciferase reporter vector (Promega). The pNGBR plasmid with PPRE deletion (pNGBR-PPRE1-del, pNGBR-PPRE2-del, pNGBR-PPREs-del) was designed and constructed using the Phusion site-directed mutagenesis kit (New England Biolabs, Ipswich, MA).

293 T cells were seeded into 48 -well plate. After reaching $\sim 90 \%$ confluence, the cells were transfected with the corresponding human pNGBR plasmid and Renilla plasmid (20:1) using Lipofectamine $^{\mathrm{TM}} 3000$ (Invitrogen, Waltham, MA, United States). After $12 \mathrm{~h}$, the cells were received the indicated treatment for $24 \mathrm{~h}$ in serum-free medium. Then, the cells were harvested and used to determine Firefly and Renilla luciferase activity with the Dual-Luciferase Reporter Assay System (Promega, Madison, WI, United States) as described (Yu et al., 2016).

\section{Chromatin Immunoprecipitation (ChIP)}

\section{Assay}

After the indicated treatment, the HUVECs were cross-linked in $1 \%$ formaldehyde solution, then cellular nuclear protein was extracted using the commercial kit (Keygen Biotech, Nanjing, China). ChIP assay was performed as previously described (Yu et al., 2020). The sequences of primers used in PCR as follows: PPRE1 forward, 5'-ATCGCATTGAAGACCACGTGTG-3'; reverse, 5 -GCCCTCAGAGACACCCCCTCTC-3'; $\quad$ PPRE2 forward, 5'-GATGATAGAGGATGTAGCATG-3', reverse, 5' CAAGACTTTGACCAACCGCCGT-3'. The results were quantified with ImageJ software (National Institutes of Health, Bethesda, MD, United States).

\section{In vivo Studies With Animals}

All the animal studies were approved by the Ethics Committee of the Hefei University of Technology and conformed to the Guide for the Care and Use of Laboratory Animals published by NIH. Animal studies are reported in compliance with the ARRIVE guidelines (Kilkenny et al., 2010; Percie Du Sert et al., 2020).

Hepatic-specific PPAR $\gamma$ knockout mice with C57BL/6J background $\left(\mathrm{PPAR} \gamma^{\mathrm{LKO}}\right)$ and the littermate control mice $\left(\mathrm{PPAR} \gamma^{\text {flox/flox }}\right.$ ) was acquired as described previously (Yang et al., 2015).

PPAR $\gamma^{\text {LKO }}$ mice and PPAR $\gamma^{\text {flox/flox }}$ mice aged $\sim 8$ weeks were divided into four groups $(n=6)$. Then the mice were treated with pioglitazone $(30 \mathrm{mg} / \mathrm{kg}$ bodyweight) or corn oil i.g. for 5 days as previously described (Yu et al., 2020). Then, all mice were euthanized by i.p injection of an overdose of pentobarbital (500 mg/kg). Liver samples were collected.

\section{Data Analysis}

We repeated all the experiments at least three times, with representative results presented. Values are presented as means \pm SEM or means \pm SD. The Shapiro Wilk method was used to analyze the normal distribution of the original data, and then Levene test was performed for the homogeneity of the square difference with SPSS software. GraphPad Prism 7 was used for statistical data analysis. Student's t-test was used for two groups and one-way ANOVA followed by Bartlett's test was used for more than two groups. The difference was considered significant at ${ }^{\star} p<0.05,{ }^{* *} p<0.01$ and ${ }^{* *} p<0.001$.

\section{RESULTS}

\section{PPARy Activation Induces NGBR Expression}

To determine whether PPAR $\gamma$ activation can influence NGBR expression, we firstly treated HUVEC cells with a synthetic PPAR $\gamma$ ligand, rosiglitazone, at different concentrations for $24 \mathrm{~h}$. The PPAR $\gamma$ and NGBR protein expression levels were 
A

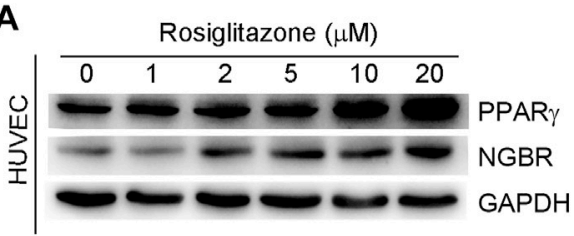

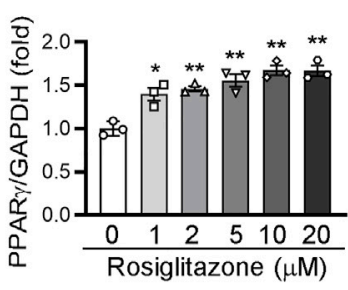

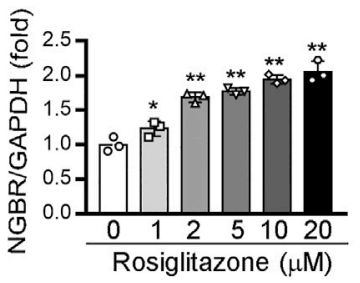

B
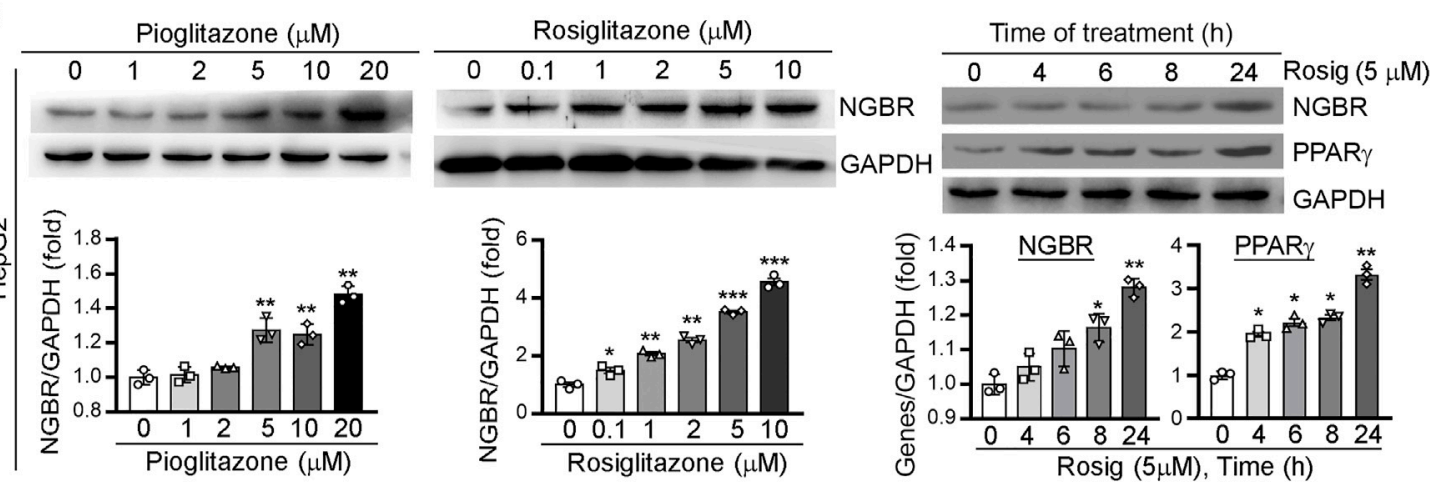

C
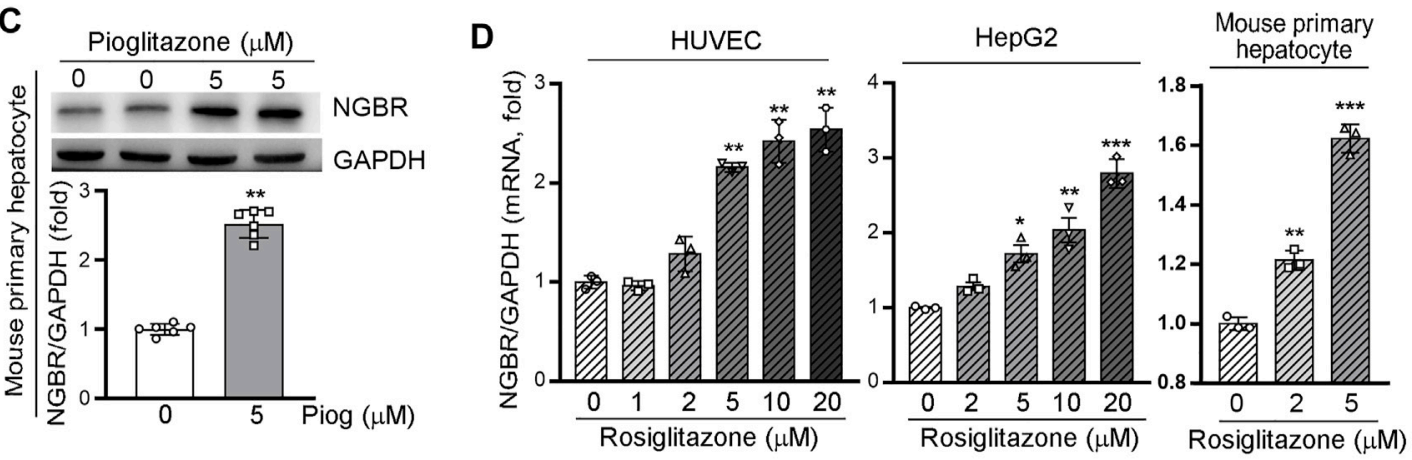

E
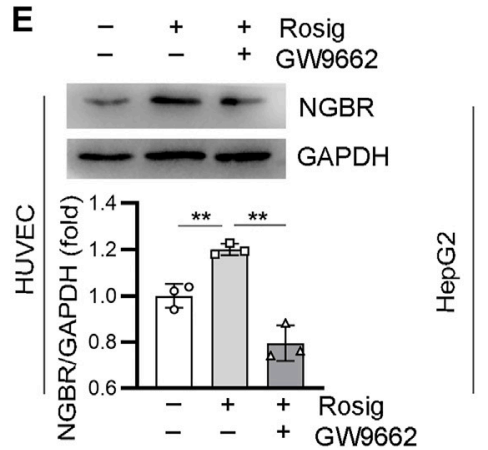

F

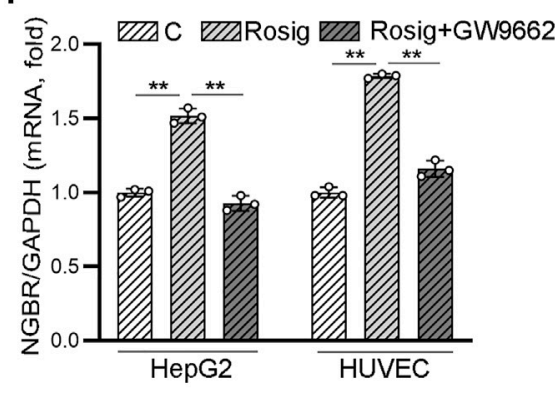

FIGURE 1 | Activation of PPAR $\gamma$ by ligand induces NGBR expression. (A-D) HUVECs, HepG2 cells and mouse primary hepatocytes were treated with rosiglitazone or pioglitazone at the indicated concentrations for $24 \mathrm{~h}$ or pioglitazone $(5 \mu \mathrm{M})$ for the indicated times. (E,F) HUVECs or HepG2 cells were treated with rosiglitazone $(10 \mu \mathrm{M})$ with or without GW9662 (10 $\mu \mathrm{M})$ for $24 \mathrm{~h}$. Levels of PPAR $\gamma$ and NGBR proteins and mRNA was determined by Western blot (A-C,E) or qPCR (D,F), respectively. Values were expressed as means $\pm \mathrm{SD},{ }^{\star} p<0.05 ;{ }^{* \star} p<0.01 ;{ }^{\star \star \star} p<0.001$ vs control $(n=3)$.

detected by Western blot. As expected, PPAR $\gamma$ was increased by rosiglitazone treatment. In line with increased PPAR $\gamma$ expression, NGBR protein expression was increased by rosiglitazone in a concentration-dependent manner (Figure 1A). Meanwhile, we observed similar results in HepG2 cells and mouse primary hepatocytes with PPAR $\gamma$ ligand treatment (Figures 1B,C). The induction of NGBR expression by rosiglitazone occurred quickly after treatment, and the maximal induction was observed at $24 \mathrm{~h}$ after treatment (right panel, Figure 1B). With the increase in protein levels, rosiglitazone also increased NGBR mRNA expression (Figure 1D), suggesting PPAR $\gamma$ activates the expression of NGBR at the transcriptional level. Moreover, we 

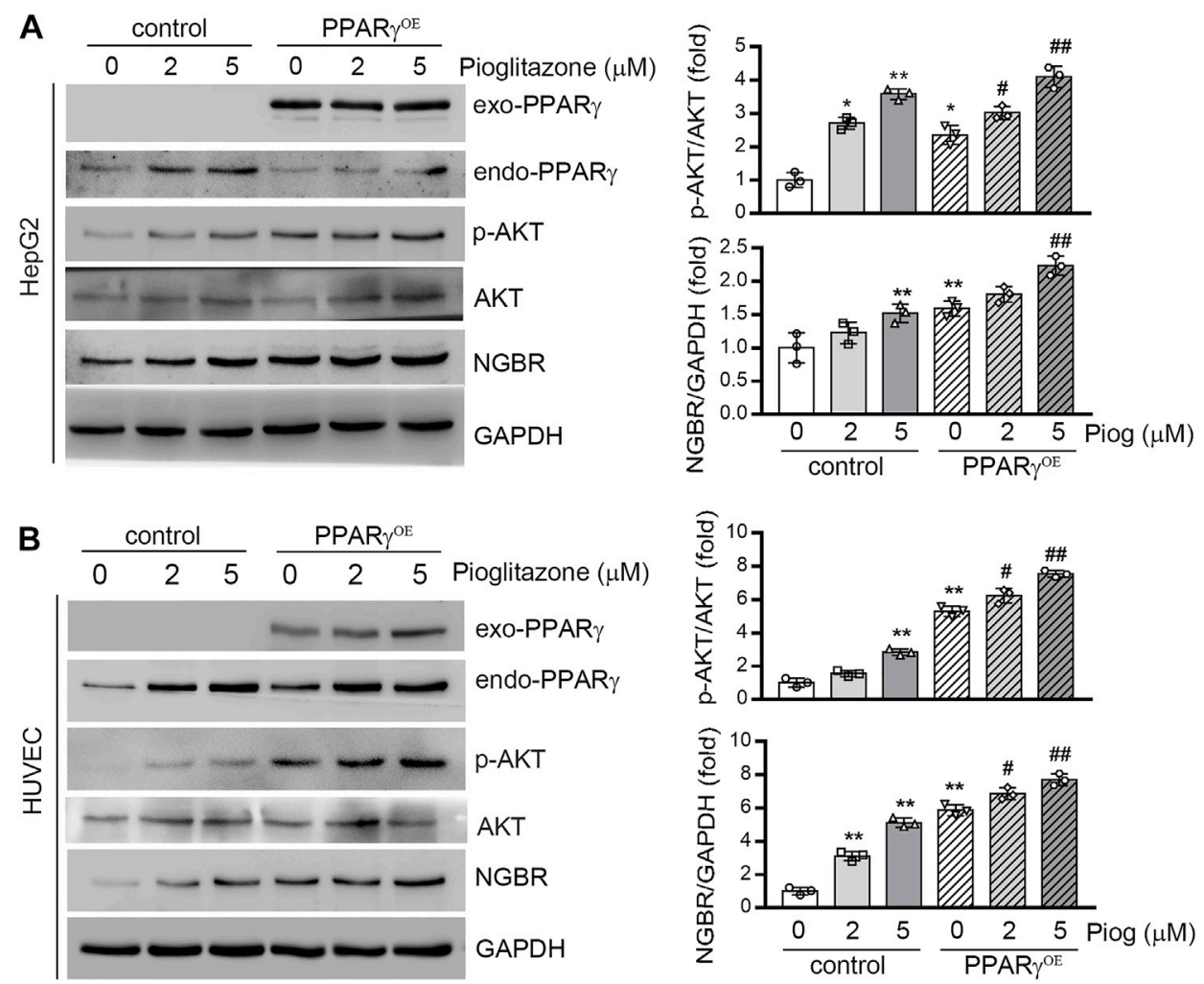

FIGURE 2 | Overexpression of PPAR $\gamma$ increases NGBR expression. HepG2 cells (A) or HUVEC (B) were transfected with the control vector (pEGFP-C2) or PPAR $\gamma$ overexpression vector ( $\mathrm{pEGFP-PPAR} \gamma$, PPAR $\gamma^{\mathrm{OE}}$ ) for $24 \mathrm{~h}$. The cells were then treated with pioglitazone at the indicated concentrations for $24 \mathrm{~h}$. Expression of $\mathrm{NGBR}$, endo-PPAR $\gamma$ and exo-PPAR $\gamma, p$-AKT and AKT was determined by Western blot. Values were expressed as means \pm SD, ${ }^{*} p<0.05$; ${ }^{* *} p<0.01$ vs control group $(n=3)$; $\# p<0.05 ; \#$; $<0.01$ vs PPAR $\gamma^{\mathrm{OE}}$ (lone group) $(n=3)$.

found that rosiglitazone-stimulated NGBR protein and mRNA expression in HUVECs and HepG2 cells were significantly reduced by the PPAR $\gamma$-specific blocker GW9662 (Figures $\mathbf{1 E}, \mathbf{F})$, indicating these effects on NGBR expression were mediated by the PPAR $\gamma$ signaling pathway.

To further confirm that PPAR $\gamma$ induced NGBR expression, we transfected HepG2 cells and HUVEC with a PPAR $\gamma$ overexpression vector. As shown in Figure 2, PPAR $\gamma$ ligandincreased NGBR expression was further enhanced by PPAR $\gamma$ overexpression. Similar to the expression of NGBR, pioglitazone and PPAR $\gamma$ overexpression enhanced AKT phosphorylation synergistically, suggesting that $\operatorname{PPAR} \gamma$ may improve insulin sensitivity via regulating NGBR expression.

\section{Identification of PPRE in NGBR Promoter}

The above results suggest PPAR $\gamma$ may activate NGBR expression at the transcriptional level. Therefore, we constructed an $1152 \mathrm{bp}$ (from -983 to +167 ) human NGBR promoter. We observed that rosiglitazone significantly activated NGBR promoter activity, and the promoter activity was gradually increased with increased rosiglitazone concentration (Figure 3A). We discovered two putative PPRE motifs in the NGBR promoter by sequence alignment analysis, which were defined as PPRE1 (from -201 to -189)and PPRE2 (from -920 to -908). To identify which PPRE plays a role in PPAR $\gamma$-induced NGBR transcription, we constructed human NGBR promoters without PPRE1 or PPRE2 or both PPREs (Figure 3B). The results in Figure 3C showed that rosiglitazone activated NGBR promoter with PPRE1 or PPRE2 deletion, while the activation was blocked with both PPREs deletion, indicating both PPREs were necessary for PPAR $\gamma$ ligand-induced NGBR expression. Furthermore, we determined that rosiglitazone enhanced the binding of PPAR $\gamma$ with both PPREs in NGBR promoter by ChIP assay (Figure 3D). Taken together, the results in Figure 3 demonstrate that NGBR is a PPAR $\gamma$ target gene, and activation of PPAR $\gamma$ induces NGBR expression at the transcriptional level.

\section{PPARy Activation Induces NGBR Expression in vivo}

To determine if activation of PPAR $\gamma$ can enhance NGBR production in vivo, PPAR $\gamma^{\mathrm{fl} / \mathrm{fl}}$ and $\mathrm{PPAR} \gamma^{\mathrm{LKO}}$ mice were treated with pioglitazone for 5 days. Then the liver was collected to determine NGBR expression. In the liver of $\operatorname{PPAR} \gamma^{\mathrm{fl} / \mathrm{fl}}$ mice pioglitazone increased NGBR expression, which was associated with increased AKT phosphorylation. In 


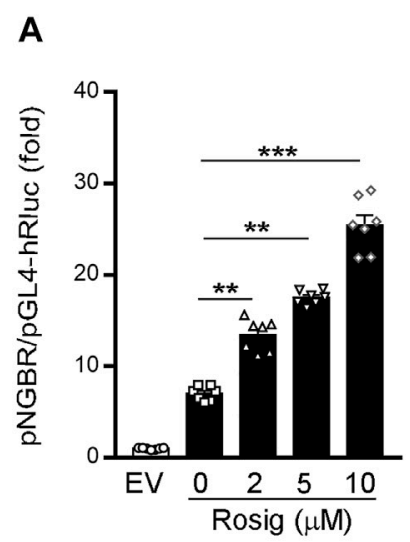
B PPRE2(-920 -908) PPRE1(-201 -189)
--TACCAAGTCGgGAATCAGGAA--AGACAAGTCCcGGGTCGTTCG-- Sequences of PPREs

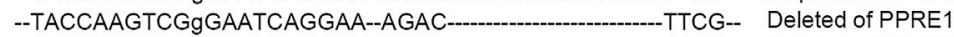

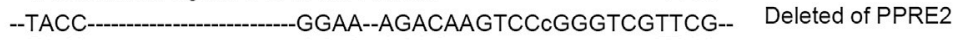

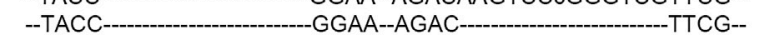
Deleted of PPREs

C

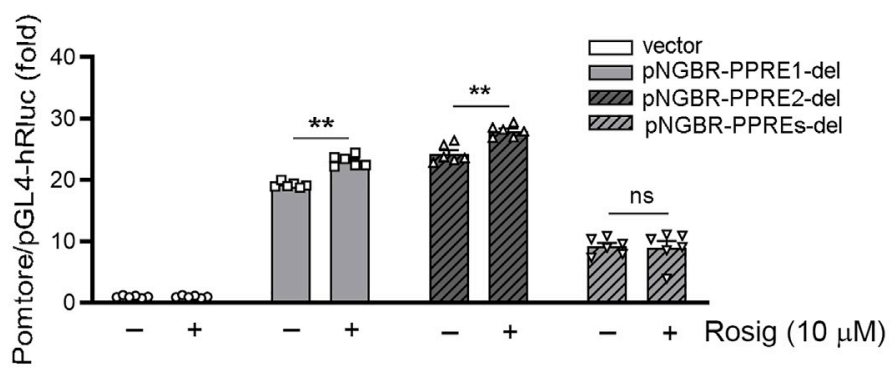

D
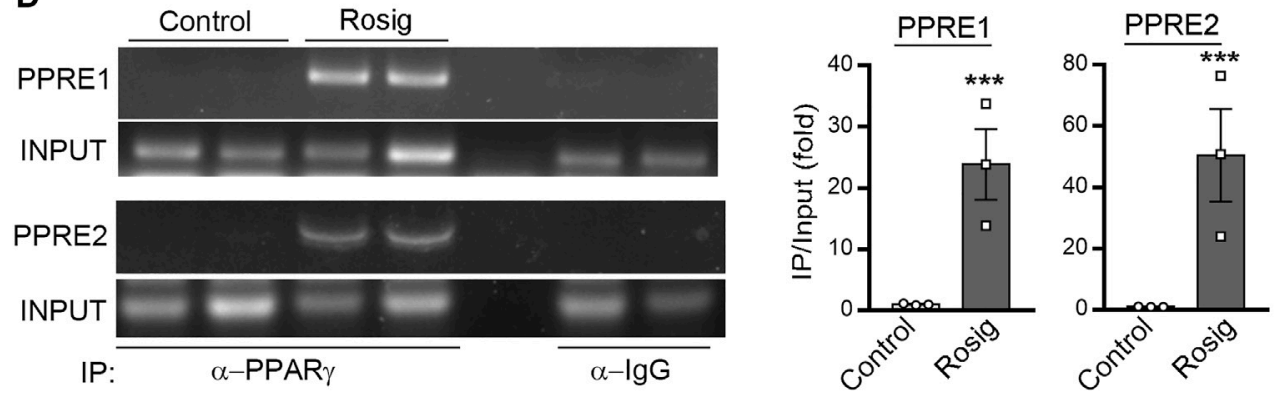

FIGURE 3 |PPAR $\gamma$ activates NGBR transcription. (A) 293T cells were transfected with pNGBR (from -983 to +167 ) and Renila luciferase vector (20:1) overnight, then treated with rosiglitazone at the indicated concentrations. ${ }^{*} p<0.05(n=7)$; (B) the sequence of NGBR promoter with the two potential PPREs (PPRE1, from -201 to -189; PPRE2, from -920 to -908), individual or both deletion; (C) 293T cells were transfected with pGL4 vector, pNGBR-PPRE1-del, pNGBR-PPRE2-del or pNGBRPPREs-del overnight. Then, the cells received the indicated treatment (rosiglitazone, $10 \mu \mathrm{M}$ ) for $24 \mathrm{~h}$ in serum-free medium. Cell lysates were used to determine firefly and Renilla luciferases activity. ${ }^{\star} p<0.05$; ${ }^{\star \star} p<0.01$; ${ }^{\star \star \star} p<0.001$; ns, not significant $(n=6)$; (D) HUVECs were treated with or without rosiglitazone (10 $\mu$ M) for 24 h. Immunoprecipitation (IP) assay was performed with anti-PPAR $\gamma$ antibody or non-specific lgG overnight. The enrichment of PPAR $\gamma$ protein in the PPRE in NGBR promoter was determined by PCR. ${ }^{\star} p<0.05 ;{ }^{\star \star} p<0.01$; ${ }^{\star \star \star} p<0.001(n=3)$. Values were expressed as means \pm SEM.

contrast, pioglitazone had little effect on hepatic NGBR expression and AKT phosphorylation in PPAR $\gamma^{\mathrm{LKO}}$ mice (Figure 4A). At the same time, by isolating mouse primary hepatocytes and treating them with pioglitazone, we found NGBR protein and mRNA expression were increased with pioglitazone in primary hepatocytes sourced from $\operatorname{PPAR} \gamma^{\mathrm{fl} / \mathrm{fl}}$ mice (Figures 4B,C).

\section{PPAR $\gamma$ Attenuates ER Stress and Inflammation via Induction of NGBR Expression}

ER stress and inflammation are observed in various pathologic situations. Our lab previously demonstrated that the knockdown of NGBR in HepG2 cells increased ER stress (Chen et al., 2021). PPAR $\gamma$ knockout $\left(\mathrm{PPAR} \gamma^{-/-}\right)$mice are developed spontaneous chronic inflammation in lung (Malur et al., 2009). Therefore, we hypothesized that PPAR $\gamma$-alleviated ER stress and inflammation may be related to its induction of NGBR expression.

To assess the involvement of NGBR on PPAR $\gamma$-reduced inflammation triggered by LPS, we transfected HUVEC with
NGBR siRNA. Knockdown of NGBR expression by siRNA increased basal level of IL- $1 \beta$, TNF- $\alpha$ and NF- $\kappa B$ (Figures 5A,B). However, the inhibitory effects of rosiglitazone on LPSinduced IL- $1 \beta$, TNF- $\alpha$ and NF- $\kappa B$ expression were abolished in NGBR siRNA-transfected cells. Interestingly, we determined that IL- $1 \beta$ and NF- $\kappa B$ expression were markedly increased in liver of PPAR $\gamma$ LKO mice. The expression of IL- $1 \beta$ and NF- $\kappa B$ reduced by pioglitazone was diminished in PPAR $\gamma^{\mathrm{LKO}}$ mice (Figure 5C). These results indicate that the reduction of inflammation by PPAR $\gamma$ was related to NGBR induction.

Similar to our previous study, knockdown of NGBR in HUVECs increased BIP and CHOP expression. Meanwhile, the inhibitory effects of rosiglitazone on tunicamycin-induced BIP protein and mRNA expression were abolished in NGBR siRNA-transfected cells (left panel, Figure 6A; middle panel, Figure 6C). In comparison, rosiglitazone-reduced $\mathrm{CHOP}$ expression was significantly attenuated in NGBR knockdown cells (middle panel, Figure 6A; left panel, Figure 6C). Moreover, we determined that the phosphorylation levels of PERK and IRE1a (p-PERK, p-IRE1a) as well as the cleavage of ATF6 (c-ATF6) were increased in NGBR knockdown cells 

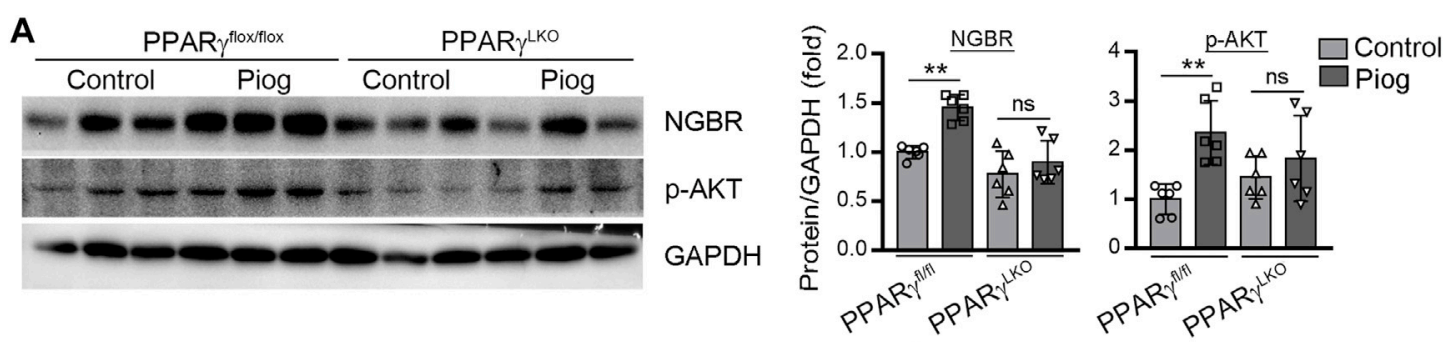

B
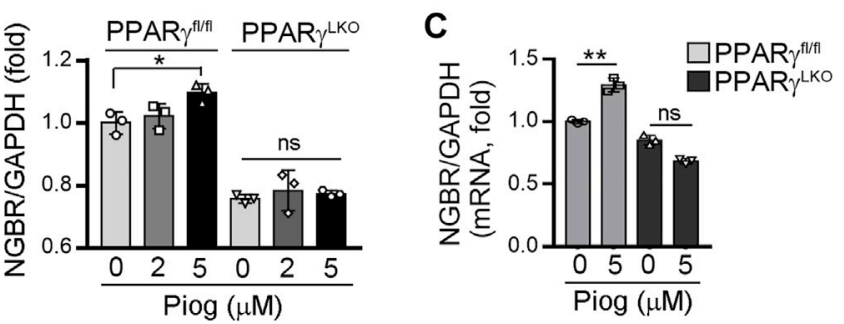

FIGURE 4 | PPAR $\gamma$ plays a significant role in pioglitazone-activated NGBR expression in vivo. (A) Pioglitazone (30 mg/kg) or corn oil was i.g. administrated with PPAR $\gamma^{\text {flox/flox }}$ and PPAR $\gamma$ LKO mice for 5 days. After treatment, liver total proteins were collected. Expression of NGBR and $p$-AKT in mouse liver was determined by Western blot. Values were expressed as means \pm SD. ${ }^{*} p<0.05$; ns, not significant $(n=6)$; (B, C) mouse primary hepatocytes treated with vehicle or pioglitazone (10 $\left.\mu \mathrm{M}\right)$ for $24 \mathrm{~h}$. The expression of NGBR protein and mRNA was determined by Western blot (B) or qPCR (C), respectively. Values were expressed as means \pm SD. ${ }^{\star} p<0.05 ;{ }^{* *} p<0.01 ;$ ns, not significant $(n=3)$.

(Figure 6B). Meanwhile, the inhibitory effects of rosiglitazone on tunicamycin-induced p-PERK, p-IRE1a and c-ATF6 were significantly attenuated in NGBR siRNA-transfected cells (Figure 6B). In vivo, the expression of BIP and CHOP was increased in the liver of PPAR $\gamma^{\mathrm{LKO}}$ mice. In PPAR $\gamma^{\mathrm{fl} / \mathrm{fl}}$ mice, pioglitazone had little effect on BIP and CHOP expression which could be attributed to the low expression of those proteins in the basal level (Figure 6D). Interestingly, pioglitazone had no effect on BIP and CHOP expression in PPAR $\gamma^{\mathrm{LKO}}$ mice (Figure 6D). Taken together, these results suggest that rosiglitazone can reduce ER stress in HUVEC and mouse liver by activating PPAR $\gamma$ NGBR pathway.

\section{DISCUSSION}

NGBR has been proved involved in many pathophysiological processes. For instance, the transduction of adenovirus NGBR can reduce free cholesterol levels and increase NPC2 levels. Mechanistic data demonstrated that the critical role of NGBR in modulating cholesterol trafficking via binding to NPC2 (Harrison et al., 2009). Interestingly, the liver-specific loss of NGBR led to increased FFA and TG levels, which was related to the activation of liver X receptor (LXR) in an NPC2-independent manner (Hu et al., 2016). We previously found that statins reduced oxysterol production and activated NGBR expression, which worked cooperatively to inactivate LXRa and restrict NAFLD (Zhang et al., 2018).

The regulation of NGBR expression and the involved mechanisms have not been fully clarified. Firstly, we observed PPAR $\gamma$ activation-induced NGBR expression in HUVEC, HepG2 cells, and mouse primary hepatocytes (Figures 1, 2).
Rosiglitazone-stimulated expression of NGBR was abolished by GW9662 (Figures 1E,F). Then, we found two PPREs in the NGBR promoter region (Figure 3B). We disclosed that PPAR $\gamma$ activated NGBR expression at the transcriptional level by promoter assay and CHIP assay (Figure 3). Finally, we found that lacking PPAR $\gamma$ expression in mouse liver abolished rosiglitazone-induced NGBR expression by using PPAR $\gamma^{\mathrm{LKO}}$ mice (Figure 4). Functionally, we found that PPAR $\gamma$-induced NGBR expression may be one of the underlying mechanisms by which PPAR $\gamma$ activation attenuated ER stress and inflammation.

Thiazolidinediones (TZDs) are insulin sensitizers and effective agonists of PPAR $\gamma$ used against T2D to increase insulin sensitivity. Insulin resistance and endothelial dysfunction are fundamental features of most patients with T2D (Steinberg et al., 1996; Ross, 1999; Tontonoz and Spiegelman, 2008). T2D is associated with elevated plasma FFA and the inappropriate deposition of lipids in the liver and skeletal muscle other than fat (Tontonoz and Spiegelman, 2008). FFAs are closely associated with impaired vascular reactivity, a measure of endothelial dysfunction. A study has showed that skeletal muscle insulin resistance may be caused by impaired insulin signaling pathways in endothelial cells (Kubota et al., 2011). Therefore, the accumulation of FFA and TG in muscle and vascular cells is closely related to insulin resistance and compromises systemic glucose disposal (Tontonoz and Spiegelman, 2008). Rosiglitazone improves systemic insulin sensitivity by activating AMPK and enhancing skeletal muscle glucose uptake (Fryer et al., 2002). In this regard, we previously determined that liver NGBR specific knockout was associated with insulin resistance and loss of $\beta$-cells in pancreas (Chen et al., 2021). Conversely, overexpression of NGBR in the liver improved insulin sensitivity by activating AMPKa and insulin signaling pathways (Chen et al., 2021). In the current study, we 

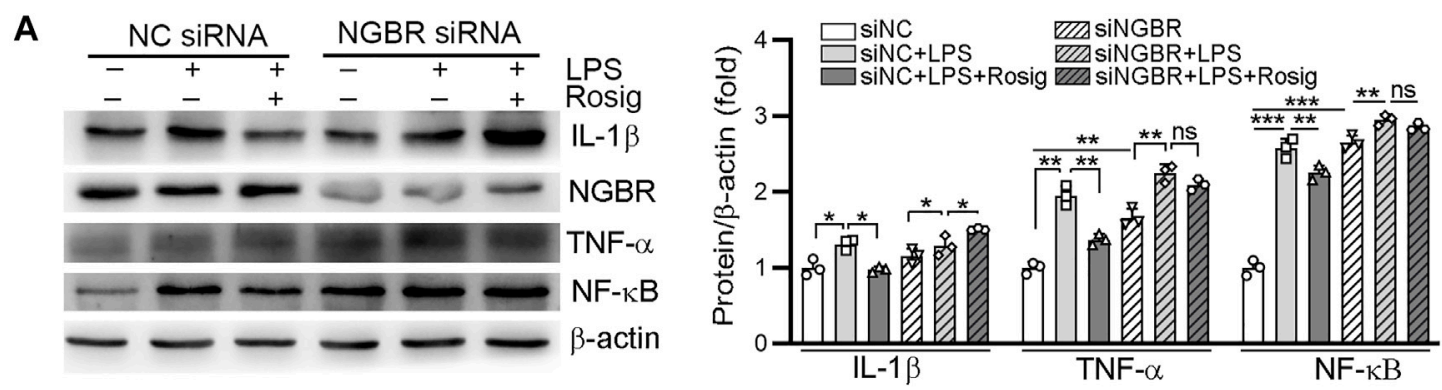

B

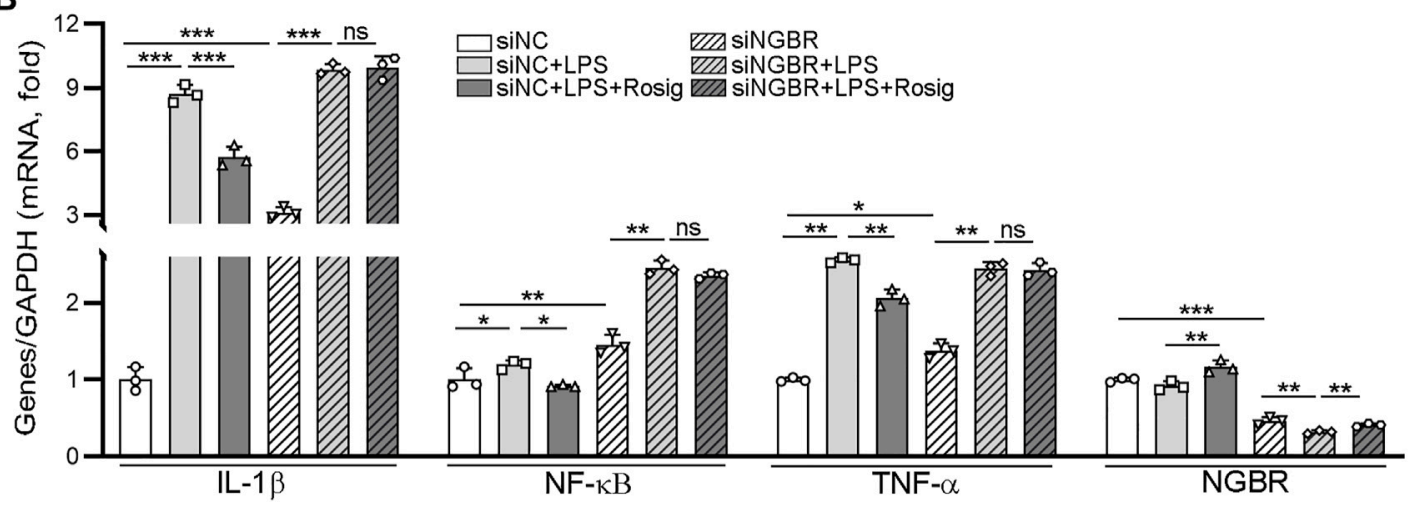

C
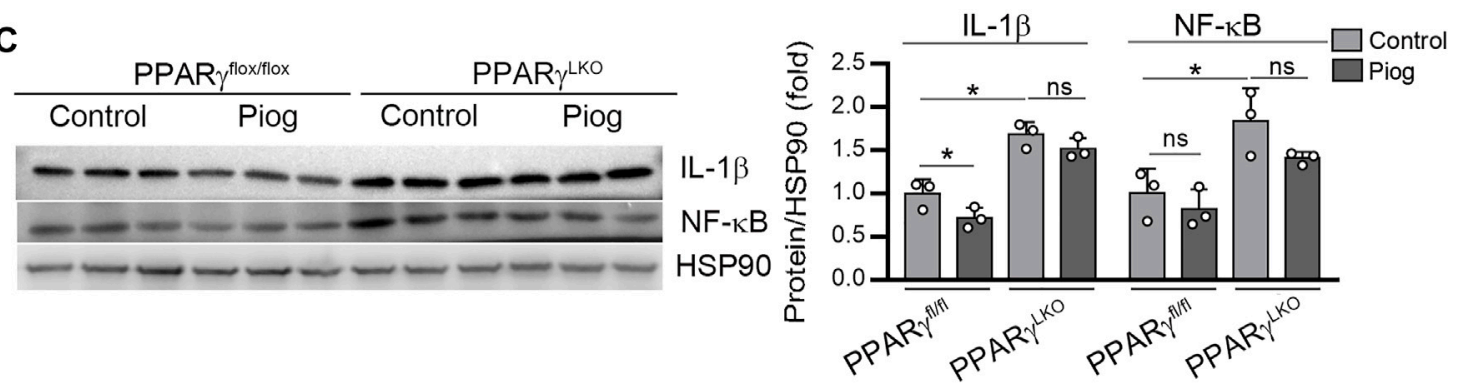

FIGURE 5|PPAR $\gamma$ attenuates LPS-induced inflammation by regulating NGBR. (A, B) HUVEC in a six-well plate were transfected with control siRNA (NC siRNA) or NGBR siRNA for $24 \mathrm{~h}$ in serum-free medium, followed by switching the cells into complete medium to culture for another $24 \mathrm{~h}$. After treatment with rosiglitazone (10 $\mu \mathrm{M}$ ) for $12 \mathrm{~h}$, all the cells were added with LPS (100 ng/ml) in the presence or absence rosiglitazone for another $12 \mathrm{~h}$. Expression of IL-1 $\beta$, TNF- $\alpha$, NF- $\kappa B$ and NGBR protein and mRNA was determined by Western blot (A) or qPCR (B), respectively. Values were expressed as means \pm SD. ${ }^{*} p<0.05$; ${ }^{\star \star} p<0.01$; ns, not significant $(n=3)$. (C) liver total proteins were collected from Figure 4. Expression of IL-1 $\beta$ and NF-kB in mouse liver was determined by Westem blot. Values were expressed as means \pm SD. ${ }^{*} p<0.05$; ns, not significant $(n=3)$.

found PPAR $y$ activation increased NGBR expression, which was related to enhanced AKT phosphorylation (Figures 2, 4A), indicating the PPAR $\gamma$-enhanced insulin sensitivity may relate to its induction of NGBR expression.

ER stress interplays with many different inflammatory and stress signaling pathways to interfere with insulin signaling (Hotamisligil, 2010). The inflammatory cytokines, such as interleukin-1 $\beta$ (IL-1 $\beta$ ) which is primarily driven by NF- $\kappa B$ and/ or NLRP3 inflammasome activation, were related to islet inflammation and insulin secretion as well as the development of insulin resistance (Boni-Schnetzler and Donath, 2011). Interestingly, NF- $\kappa \mathrm{B}$ and/or NLRP3 inflammasome can be activated directly by ER stress in cultured islets (Hotamisligil, 2010; Lerner et al., 2012). Many studies have shown PPAR $\gamma$ agonists reduce inflammation in multiple immune cells
(Bessueille and Magne, 2015). For instance, PPAR $\gamma$ regulates macrophage polarization toward an anti-inflammatory phenotype (M2) (Duan et al., 2009). The differentiation of M2 macrophages results in increased expression of PPAR $\gamma$. In contrast, PPAR $\gamma$ agonists inhibit the M1 phenotype and decrease the expression of inflammatory cytokines, such as TNF- $\alpha$, IL- $1 \beta$, and IL-6 (Berry et al., 2007). Moreover, PPAR $\gamma$ regulates the inflammatory involvement of the immunogenicity of dendritic cells observed in atherosclerosis (Nencioni et al., 2002). Mechanistically, PPAR $\gamma$ represses the transcriptional activation of inflammatory response genes via SUMOylation of the PPAR $\gamma$ ligand-binding domain. SuMOylated PPAR $\gamma$ stabilized nuclear receptor corepressor-histone deacetylase-3 complexes within the promoter region of most inflammatory genes, which represses those genes expression (Pascual et al., 2005). Herein, we 
A

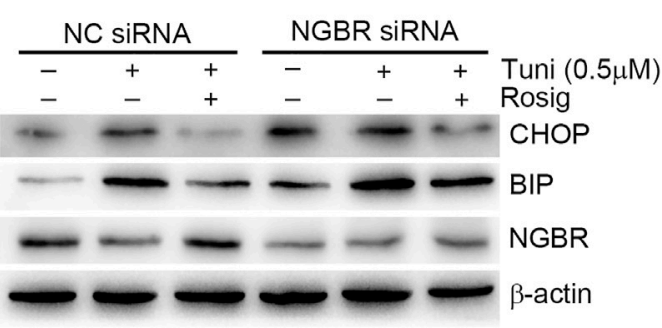

B

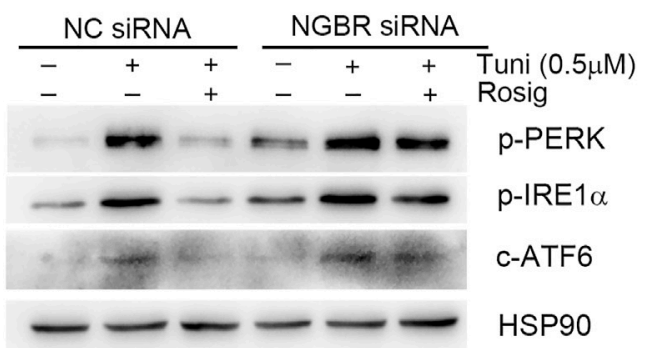

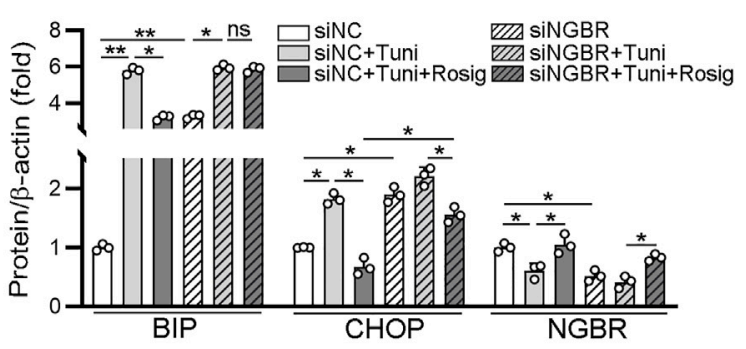

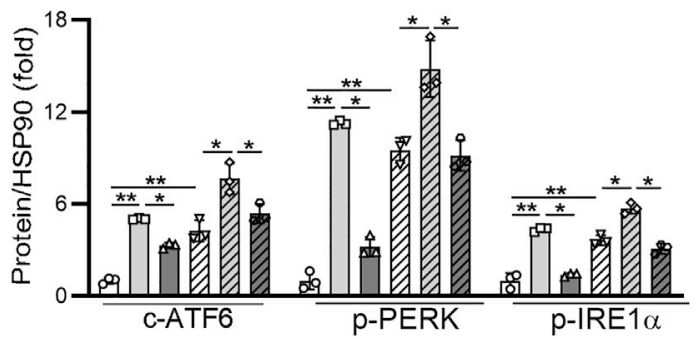

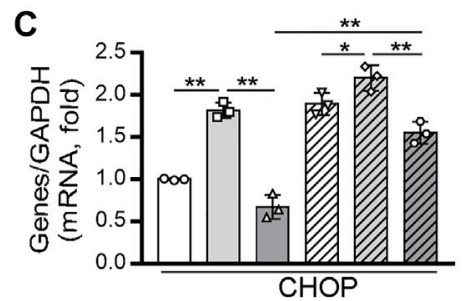
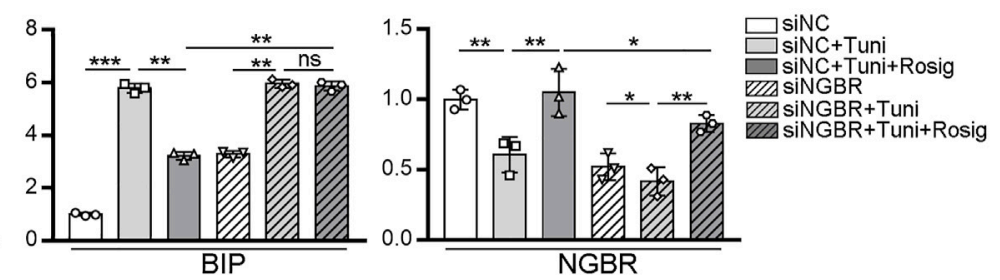

D
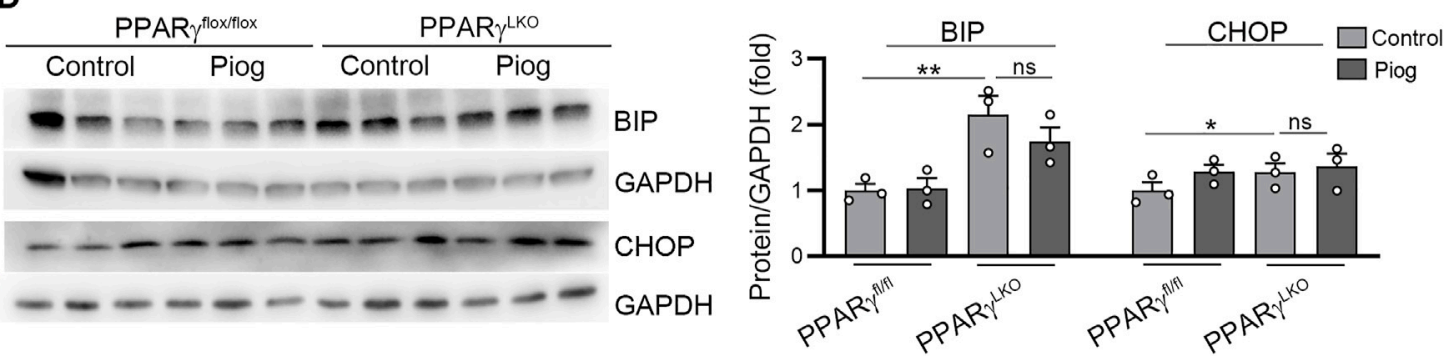

FIGURE 6 | PPAR $\gamma$ reduces tunicamycin-induced ER stress by regulating NGBR. (A-C) HUVEC cells in a six-well plate were transfected with control siRNA (NC siRNA) or NGBR siRNA for $24 \mathrm{~h}$ in serum-free medium, followed by switching the cells into complete medium to culture for another $24 \mathrm{~h}$. After treatment with rosiglitazone $(10 \mu \mathrm{M})$ for $12 \mathrm{~h}$, the cells were treated with tunicamycin $(0.5 \mu \mathrm{g} / \mathrm{ml})$ with or without rosiglitazone for another $12 \mathrm{~h}$. Expression of $\mathrm{CHOP}$, BIP, NGBR p-PERK, p-IRE1 $\alpha$ and c-ATF6 protein was determined by Western blot (A, B). Expression of CHOP, BIP and NGBR mRNA was determined by qPCR (C). Values were expressed as means \pm SD. ${ }^{\star} p<0.05 ;{ }^{\star \star} p<0.01$; ns, not significant $(n=3)$. (D) liver total proteins were collected from Figure 4. Expression of BIP and CHOP in mouse liver was determined by Western blot. Values were expressed as means \pm SD. ${ }^{*} p<0.05 ;{ }^{* *} p<0.01$; ns, not significant $(n=3)$.

determined that PPAR $\gamma$ ligand rosiglitazone reduced LPS-induced inflammation in control HUVECs, which was abolished in NGBR siRNA transfected cells (Figure 5), suggesting that NGBR is an important mediator involved in PPAR $\gamma$-inhibited inflammation.

In conclusion, our study shows that NGBR is a new molecular target of PPAR $\gamma$ and demonstrates that PPAR $\gamma$ attenuates ER stress and inflammation partly through the induction of NGBR expression. Our study also suggests that PPAR $\gamma$ activation may enhance insulin sensitivity via regulating NGBR expression.

\section{DATA AVAILABILITY STATEMENT}

The original contributions presented in the study are included in the article/supplementary material, further inquiries can be directed to the corresponding author.

\section{ETHICS STATEMENT}

The animal study was reviewed and approved by Ethics Committee of the Hefei University of Technology. 


\section{AUTHOR CONTRIBUTIONS}

JM, PZ, LL carried out the experiments; MZ, JZ, CW, and XZ analyzed data; $\mathrm{QM}, \mathrm{WH}, \mathrm{YD}, \mathrm{XY}$ and $\mathrm{JH}$ provided valuable suggestions and revised the manuscript. YC designed the experiments and wrote the paper.

\section{REFERENCES}

Berry, A., Balard, P., Coste, A., Olagnier, D., Lagane, C., Authier, H., et al. (2007). IL-13 Induces Expression of CD36 in Human Monocytes Through PPARgamma Activation. Eur. J. Immunol. 37, 1642-1652. doi:10.1002/ eji.200636625

Bessueille, L., and Magne, D. (2015). Inflammation: a Culprit for Vascular Calcification in Atherosclerosis and Diabetes. Cell Mol Life Sci. 72, 2475-2489. doi:10.1007/s00018-015-1876-4

Böni-Schnetzler, M., and Donath, M. Y. (2011). Increased IL-1 $\beta$ Activation, the Culprit Not Only for Defective Insulin Secretion but Also for Insulin Resistance? Cell Res. 21, 995-997. doi:10.1038/cr.2011.85

Chen, Y., Duan, Y., Kang, Y., Yang, X., Jiang, M., Zhang, L., et al. (2012). Activation of Liver X Receptor Induces Macrophage Interleukin-5 Expression. J. Biol. Chem. 287, 43340-43350. doi:10.1074/jbc.M112.403394

Chen, Y., Hu, W., Li, Q., Zhao, S., Zhao, D., Zhang, S., et al. (2021). NGBR Is Required to Ameliorate Type 2 Diabetes in Mice by Enhancing Insulin Sensitivity. J. Biol. Chem. 296, 100624. doi:10.1016/j.jbc.2021.100624

Duan, S. Z., Usher, M. G., and Mortensen, R. M. (2009). PPARs: the Vasculature, Inflammation and Hypertension. Curr. Opin. Nephrol. Hypertens. 18, 128-133. doi:10.1097/MNH.0b013e328325803b

Duan, Y., Chen, Y., Hu, W., Li, X., Yang, X., Zhou, X., et al. (2012). Peroxisome Proliferator-Activated Receptor $\gamma$ Activation by Ligands and Dephosphorylation Induces Proprotein Convertase Subtilisin Kexin Type 9 and Low Density Lipoprotein Receptor Expression. J. Biol. Chem. 287, 23667-23677. doi:10.1074/jbc.M112.350181

Fryer, L. G., Parbu-Patel, A., and Carling, D. (2002). The Anti-diabetic Drugs Rosiglitazone and Metformin Stimulate AMP-Activated Protein Kinase through Distinct Signaling Pathways. J. Biol. Chem. 277, 25226-25232. doi:10.1074/jbc.M202489200

Fu, S., Watkins, S. M., and Hotamisligil, G. S. (2012). The Role of Endoplasmic Reticulum in Hepatic Lipid Homeostasis and Stress Signaling. Cell Metab. 15, 623-634. doi:10.1016/j.cmet.2012.03.007

Harrison, K. D., Miao, R. Q., Fernandez-Hernándo, C., Suárez, Y., Dávalos, A., and Sessa, W. C. (2009). Nogo-B Receptor Stabilizes Niemann-Pick Type C2 Protein and Regulates Intracellular Cholesterol Trafficking. Cell Metab. 10, 208-218. doi:10.1016/j.cmet.2009.07.003S1550-4131(09)00199-5

Hetz, C. (2012). The Unfolded Protein Response: Controlling Cell Fate Decisions Under ER Stress and beyond. Nat. Rev. Mol. Cell Biol. 13, 89-102. doi:10.1038/ nrm 3270

Hotamisligil, G. S. (2010). Endoplasmic Reticulum Stress and the Inflammatory Basis of Metabolic Disease. Cell. 140, 900-917. doi:10.1016/j.cell.2010.02.034

Hu, W., Zhang, W., Chen, Y., Rana, U., Teng, R. J., Duan, Y., et al. (2016). Nogo-B Receptor Deficiency Increases Liver X Receptor Alpha Nuclear Translocation and Hepatic Lipogenesis through an Adenosine Monophosphate-Activated Protein Kinase Alpha-dependent Pathway. Hepatology. 64, 1559-1576. doi:10.1002/hep.28747

Huang, F., Zeng, Z., Zhang, W., Yan, Z., Chen, J., Yu, L., et al. (2021). Design, Synthesis, and Biological Evaluation of Novel Sulindac Derivatives as Partial Agonists of PPAR $\gamma$ with Potential Anti-Diabetic Efficacy. Eur. J. Med. Chem. 222, 113542. doi:10.1016/j.ejmech.2021.113542

Kharroubi, I., Ladrière, L., Cardozo, A. K., Dogusan, Z., Cnop, M., and Eizirik, D. L. (2004). Free Fatty Acids and Cytokines Induce Pancreatic Beta-Cell Apoptosis by Different Mechanisms: Role of Nuclear Factor-KappaB and Endoplasmic Reticulum Stress. Endocrinology. 145, 5087-5096. doi:10.1210/ en.2004-0478

Kilkenny, C., Browne, W., Cuthill, I. C., Emerson, M., Altman, D. G., and Group, N. C. R. R. G. W. (2010). Animal Research: Reporting In Vivo Experiments: the

\section{FUNDING}

This work was supported by the National Natural Science Foundation of China (NSFC) Grants 31770863 to YC, 81973316 to $\mathrm{JH}$, and 81803517 to $\mathrm{XY}$; the China Postdoctoral Science Foundation Grants 2020M681914 to XY.

ARRIVE Guidelines. J. Physiol. 588, 2519-2521. doi:10.1111/j.14765381.2010.00872.x10.1113/jphysiol.2010.192278

Kubota, T., Kubota, N., Kumagai, H., Yamaguchi, S., Kozono, H., Takahashi, T., et al. (2011). Impaired Insulin Signaling in Endothelial Cells Reduces InsulinInduced Glucose Uptake by Skeletal Muscle. Cell Metab. 13, 294-307. doi:10.1016/j.cmet.2011.01.018

Lerner, A. G., Upton, J. P., Praveen, P. V., Ghosh, R., Nakagawa, Y., Igbaria, A., et al (2012). IRE1a Induces Thioredoxin-Interacting Protein to Activate the NLRP3 Inflammasome and Promote Programmed Cell Death under Irremediable ER Stress. Cell Metab. 16, 250-264. doi:10.1016/j.cmet.2012.07.007

Malur, A., Mccoy, A. J., Arce, S., Barna, B. P., Kavuru, M. S., Malur, A. G., et al. (2009). Deletion of PPAR Gamma in Alveolar Macrophages Is Associated with a Th-1 Pulmonary Inflammatory Response. J. Immunol. 182, 5816-5822. doi:10.4049/jimmunol.0803504

Miao, R. Q., Gao, Y., Harrison, K. D., Prendergast, J., Acevedo, L. M., Yu, J., et al. (2006). Identification of a Receptor Necessary for Nogo-B Stimulated Chemotaxis and Morphogenesis of Endothelial Cells. Proc. Natl. Acad. Sci. U S A. 103, 10997-11002. doi:10.1073/pnas.0602427103

Nencioni, A., Grünebach, F., Zobywlaski, A., Denzlinger, C., Brugger, W., and Brossart, P. (2002). Dendritic Cell Immunogenicity Is Regulated by Peroxisome Proliferator-Activated Receptor Gamma. J. Immunol. 169, 1228-1235. doi:10.4049/jimmunol.169.3.1228

Pascual, G., Fong, A. L., Ogawa, S., Gamliel, A., Li, A. C., Perissi, V., et al. (2005). A SUMOylation-dependent Pathway Mediates Transrepression of Inflammatory Response Genes by PPAR-Gamma. Nature. 437, 759-763. doi:10.1038/ nature 03988

Percie Du Sert, N., Hurst, V., Ahluwalia, A., Alam, S., Avey, M. T., Baker, M., et al. (2020). The ARRIVE Guidelines 2.0: Updated Guidelines for Reporting Animal Research. J. Cereb. Blood Flow Metab. 40, 1769-1777. doi:10.1111/bph.15193

Ricote, M., Li, A. C., Willson, T. M., Kelly, C. J., and Glass, C. K. (1998). The Peroxisome Proliferator-Activated Receptor-Gamma Is a Negative Regulator of Macrophage Activation. Nature. 391, 79-82. doi:10.1038/34178

Ross, R. (1999). Atherosclerosis--an Inflammatory Disease. N. Engl. J. Med. 340, 115-126. doi:10.1056/NEJM199901143400207

Steinberg, H. O., Chaker, H., Leaming, R., Johnson, A., Brechtel, G., and Baron, A. D. (1996). Obesity/Insulin Resistance Is Associated with Endothelial Dysfunction. Implications for the Syndrome of Insulin Resistance. J. Clin. Invest. 97, 2601-2610. doi:10.1172/JCI118709

Straus, D. S., and Glass, C. K. (2007). Anti-inflammatory Actions of PPAR Ligands: New Insights on Cellular and Molecular Mechanisms. Trends Immunol. 28, 551-558. doi:10.1016/j.it.2007.09.003

Tontonoz, P., and Spiegelman, B. M. (2008). Fat and Beyond: the Diverse Biology of PPARgamma. Annu. Rev. Biochem. 77, 289-312. doi:10.1146/ annurev.biochem.77.061307.091829

Walter, P., and Ron, D. (2011). The Unfolded Protein Response: from Stress Pathway to Homeostatic Regulation. Science. 334, 1081-1086. doi:10.1126/ science. 1209038

Wang, S., and Kaufman, R. J. (2012). The Impact of the Unfolded Protein Response on Human Disease. J. Cell Biol. 197, 857-867. doi:10.1083/jcb.201110131

Wu, D., Zhao, B., Qi, X., Peng, F., Fu, H., Chi, X., et al. (2018). Nogo-B Receptor Promotes Epithelial-Mesenchymal Transition in Non-Small Cell Lung Cancer Cells through the Ras/ERK/Snail1 Pathway. Cancer Lett. 418, 135-146. doi:10.1016/j.canlet.2018.01.030

Xue, P., Li, B., An, Y., Sun, J., He, X., Hou, R., et al. (2016). Decreased MORF Leads to Prolonged Endoplasmic Reticulum Stress in Periodontitis-Associated Chronic Inflammation. Cell Death Differ. 23, 1862-1872. doi:10.1038/ cdd.2016.74

Yang, X., Yao, H., Chen, Y., Sun, L., Li, Y., Ma, X., et al. (2015). Inhibition of Glutathione Production Induces Macrophage CD36 Expression and Enhances 
Cellular-Oxidized Low Density Lipoprotein (oxLDL) Uptake. J. Biol. Chem. 290, 21788-21799. doi:10.1074/jbc.M115.654582

Yoshiuchi, K., Kaneto, H., Matsuoka, T. A., Kasami, R., Kohno, K., Iwawaki, T., et al. (2009). Pioglitazone Reduces ER Stress in the Liver: Direct Monitoring of In Vivo ER Stress Using ER Stress-Activated Indicator Transgenic Mice. Endocr. J. 56, 1103-1111. doi:10.1507/endocri.k09e-140

Yu, M., Jiang, M., Chen, Y., Zhang, S., Zhang, W., Yang, X., et al. (2016). Inhibition of Macrophage Cd36 Expression and Cellular Oxidized Low Density Lipoprotein (Oxldl) Accumulation by Tamoxifen: A Peroxisome

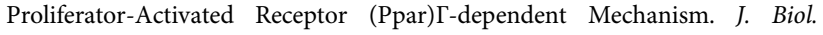
Chem. 291, 16977-16989. doi:10.1074/jbc.M116.740092

Yu, M., Zhang, S., Guo, F., Yang, X., Li, Q., Wei, Z., et al. (2020). Identification of Nogo-B as a New Molecular Target of Peroxisome Proliferator-Activated Receptor Gamma. Cell Signal. 65, 109429. doi:10.1016/j.cellsig.2019.109429

Zhang, W., Yang, X., Chen, Y., Hu, W., Liu, L., Zhang, X., et al. (2018). Activation of Hepatic Nogo-B Receptor Expression-A New Anti-liver Steatosis Mechanism of Statins. Biochim. Biophys. Acta Mol. Cell Biol Lipids. 1863, 177-190. doi:10.1016/j.bbalip.2017.12.002
Conflict of Interest: The authors declare that the research was conducted in the absence of any commercial or financial relationships that could be construed as a potential conflict of interest.

Publisher's Note: All claims expressed in this article are solely those of the authors and do not necessarily represent those of their affiliated organizations, or those of the publisher, the editors and the reviewers. Any product that may be evaluated in this article, or claim that may be made by its manufacturer, is not guaranteed or endorsed by the publisher.

Copyright $\odot 2022 \mathrm{Ma}$, Zeng, Liu, Zhu, Zheng, Wang, Zhao, Hu, Yang, Duan, Han, Miao and Chen. This is an open-access article distributed under the terms of the Creative Commons Attribution License (CC BY). The use, distribution or reproduction in other forums is permitted, provided the original author(s) and the copyright owner(s) are credited and that the original publication in this journal is cited, in accordance with accepted academic practice. No use, distribution or reproduction is permitted which does not comply with these terms. 